\title{
Primer registro de Pisione guanche San Martín, López \& Núñez, 1999 (Annelida: Sigalionidae) en el golfo de Vizcaya.
}

\author{
First record of Pisione guanche San Martín, López \& \\ Núñez, 1999 (Annelida: Sigalionidae) in the Bay of \\ Biscay.
}

Julián Martínez ${ }^{1}$, Idoia Adarraga' ${ }^{1}$, Mikel Aitor Marquiegui ${ }^{1}$

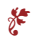

\section{Resumen}

En el transcurso de un programa de vigilancia ambiental llevado a cabo por INSUB el 9 de Mayo de 2017, dos ejemplares completos y un fragmento de Pisione guanche San Martín, López \& Núñez, 1999 (Annelida: Polychaeta: Sigalionidae) han sido identificados. Esta es la primera referencia de la especie para el golfo de Vizcaya. Los ejemplares fueron obtenidos en fondos blandos circalitorales de la plataforma continental de Zarautz (Costa vasca, SE del golfo de Vizcaya). En el presente artículo se aportan aspectos morfológicos, geográficos y ecológicos de la especie.

Palabras clave: Primer registro, Pisione guanche, Annelida, Sigalionidae, golfo de Vizcaya.

\begin{abstract}
During a monitoring programme carried out on 9 May 2017 by INSUB, two complete and one incomplete specimen of Pisione guanche San Martín, López \& Núñez, 1999 (Annelida: Polychaeta: Sigalionidae) were identified. This is the first record of the species in the Bay of Biscay. The specimens were found in circalittoral soft bottoms of the continental shelf of Zarautz (Basque coast, SE Bay of Biscay). In this paper, morphological features, distribution and ecological aspects of this species are provided.
\end{abstract}

Key words: First record, Pisione guanche, Annelida, Sigalionidae, Bay of Biscay.

\footnotetext{
1 Museo de Oquendo. Sociedad Cultural INSUB

C/ Zemoria, 12. Apdo. de Correos 3223

20013 Donostia-San Sebastián. Gipuzkoa

*Correspondencia: julidoia@outlook.com
} 


\section{Laburpena}

INSUBek 2017ko maiatzaren 9an burututako ikerketa-programa batean zehar Pisione guanche San Martín, López \& Núñez, 1999 (Annelida: Polychaeta: Sigalionidae) espeziearen bi ale oso eta osatugabeko bat identifikatu dira. Hau da Bizkaiko Golkoan * aipatzen den lehenbiziko aldia. Aleak Zarauzko (Euskal Itsasertza, Bizkaiko Golkoko SE) plataforma kontinentaleko zirkalitoraleko hondo gogorretan aurkitu ziren. Artikulu honetan P. guanche-ren ezaugarri morfologiko, geografiko eta ekologikoen ekarpena egiten da.

Gako hitzak: lehen erregistroa, Pisione guanche, Annelida, Sigalionidae, Bizkaiko Golkoa.

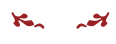

El género Pisione Grube, 1857 agrupa a un conjunto de anélidos, por lo general de pequeño tamaño, distribuido preferentemente en regiones templadas y tropicales del planeta (Salcedo et al., 2015). La posición sistemática de las especies que lo integran ha sido bastante discutida en los últimos años. Históricamente, el género Pisione se ha incluido en la familia Pisionidae junto a los géneros Pisionella Hartman, 1939, Pisionidens Aiyar \& Alikunhi, 1943 y Anoplopisione Laubier, 1967. Sin embargo estudios filogenéticos los ubican dentro de la familia Sigalionidae (Struck et al., 2005; Wiklund et al., 2005; Norlinder et al., 2012). Esta nueva posición resulta a priori sorprendente, debido a que uno de los aspectos más característicos de todos los integrantes de la familia Sigalionidae es la posesión de élitros (modificaciones de los cirros dorsales parapodiales en cierto número de segmentos) que se hallan ausentes en dichos géneros. A pesar de ello, Struck et al. (2005) y Wiklund et al. (2005) justifican su ausencia como resultado de un proceso adaptativo de los pisiónidos a su vida intersticial. A esta peculiaridad anatómica de los pisiónidos dentro de los Sigalionidae se sumaría la presencia en ellos de órganos copuladores, aspecto también atribuido con anterioridad por Laubier (1967) y Westheide (1971) a la adopción de un modo de vida entre los granos de arena.

En la actualidad se reconocen 46 especies y subespecies válidas dentro del género Pisione (Gonzalez et al., 2017), de las cuales todas, a excepción de Pisione garciavaldecasasi San Martín, López \& Camacho, 1998 que se encuentra en cursos fluviales de Panamá (San Martín et al., 1998), habitan en ambientes marinos. La mayoría se encuentran en fondos someros de escasa profundidad, si bien algunas especies pueden vivir en fondos circalitorales, a profundidades próximas a los 200 m (Yamanishi, 1998; San Martín, 2004).

En el golfo de Vizcaya únicamente tres especies de Pisione habían sido referenciadas hasta la fecha: P. remota (Southern, 1914) especie supuestamente cosmopolita con amplia representación en arenas medias y gruesas de todo el golfo de Vizcaya (Aguirrezabalaga et al., 1984); P. parapari Moreira, Quintas \& Troncoso, 2000 procedente de la Ensenada de Bayona (Pontevedra, NO península Ibérica) (Moreira et al., 2000) e identificada también en 
la costa vasca (datos no publicados); y P. inkoi Martínez, Aguirrezabalaga \& Adarraga, 2008, común en arenas gruesas circalitorales del litoral vasco (Martínez et al., 2008).

Durante el desarrollo de un estudio de vigilancia ambiental llevado a cabo en la costa de Zarautz (Guipúzcoa, SE del golfo de Vizcaya) dos ejemplares completos y un fragmento posterior de P. guanche San Martín, López \& Núñez, 1999 han sido identificados. Esta es la primera ocasión que se cita la especie para la fauna del golfo de Vizcaya, además de representar el registro más septentrional de la especie (Fig. 1).

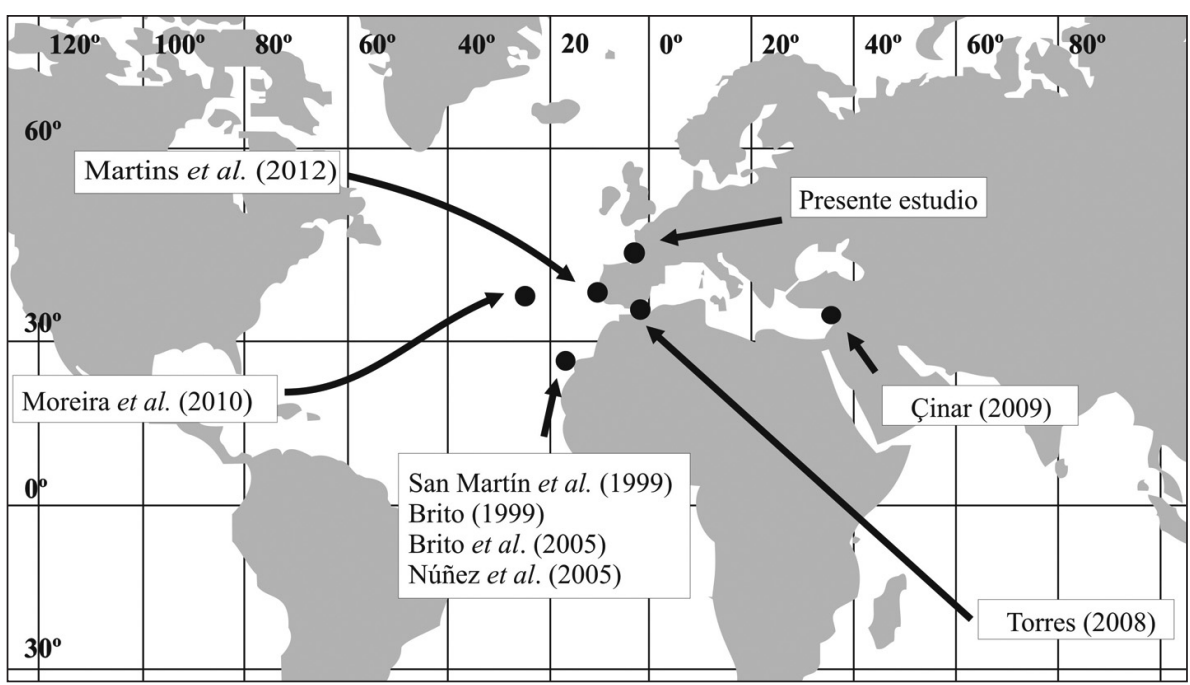

Fig. 1.- Distribución de todos los registros mundiales de Pisione guanche.

Fig. 1.- Distribution of all the records of Pisione guanche worldwide.

\section{Sistemática}

Phylum Annelida

Orden Phyllodocida Levinsen, 1883

Familia Sigalionidae Malmgren, 1867

Genus Pisione Grube, 1857

Pisione guanche San Martín, López \& Núñez, 1999

Pisione guanche San Martín, López \& Núñez, 1999: 29, figs 2 y 3; Brito, 1999: 262, figs. 63a, 64a-f; Herrando-Pérez et al., 2001: 275 (tab. 2); Brito et al., 2005: 24 (tab. 4); Núñez et al., 2005: 297 (tab. 1); Torres, 2008: 213 (pl. 5); Çinar, 2009: 4, figs. 2B-E; Moreira et al., 2010: 1, figs. 1-3; Martins et al., 2012: 12 (tab. 4), figs. 1 y 2. 


\section{Material examinado}

Zarautz, estación M5-30 (43018'18“N, 209’04“W), 2 ejemplares completos y un fragmento posterior de 33 setígeros, arenas gruesas, $31 \mathrm{~m}$ de profundidad, 09.05.2017.

Uno de los ejemplares completos así como el fragmento posterior que contiene los órganos copuladores de un macho, se han depositado en la colección del Museo Nacional de Ciencias Naturales de Madrid, España (Códigos MNCN 16.01/17823 y MNCN 16.01/17824, respectivamente).

\section{Descripción de los ejemplares de Zarautz}

Cuerpo relativamente largo y fino con numerosos segmentos (Fig. 2A). Ejemplares completos con una longitud de 11,9 y 10,2 mm, 0,6 y 0,48 mm de anchura máxima y 71 y 68 setígeros respectivamente. Prostomio pequeño, romboidal u ovalado y rodeado por el segmento bucal. Un par de palpos largos, lisos y dirigidos hacia adelante. Segmento bucal con dos lóbulos que se proyectan anteriormente; cada uno de ellos soportados por una acícula bucal oblicuamente orientada que sobresale de la abertura bucal. Acículas bucales robustas, amarillentas y extremo denticulado (Fig. 2B). Lóbulos cerebrales bilobulados posteriormente, extendiéndose hasta el final del setígero 3. Dos pares de ojos conspicuos, bien visibles dorsalmente, situados entre los setígeros 1 y 2 . Un par de cirros bucales dorsales alargados y de longitud claramente inferior a los palpos. Cirros ventrales del primer setígero alargados, sobrepasando ligeramente la base de los palpos. Probóscide muscular, provista de dos pares de mandíbulas localizadas entre el tercer y sexto setígero. Cirros dorsales (excepto los del segundo setígero) pequeños, globulares a piriformes, con una pequeña papila distal y extremo irregularmente piloso. Cirros dorsales del segundo setígero dos a tres veces más largos que los restantes (Fig. 2C). Cirros ventrales (excepto los del primer setígero) similares en forma y tamaño a los dorsales. Parápodos alargados, truncados y con el lóbulo presetal bilobulado (Fig. 2D,E). Dos acículas por parápodo; la superior más robusta, sobresale del lóbulo parapodial a partir del octavo o noveno setígero. Unas cinco sedas por parápodo, incluyendo una (ocasionalmente dos) seda simple dorsal y cuatro sedas compuestas heterogonfas falcígeras (Fig. 2F). Seda simple dorsal robusta, con el extremo truncado oblicuamente y espinas distales más conspícuas en los setígeros anteriores (Fig. 2G); sedas compuestas heterogonfas con los artejos falcígeros cortos, unidentados y espinas marginales; artejos en cada uno de los parápodos con gradación dorsal en su longitud, más aparente en los setígeros anteriores. En los setígeros posteriores estos artejos se muestran cada vez más cortos (Fig. 2H,I). Pigidio bilobulado con dos cirros cirros anales largos.

Aparato reproductor masculino presente en un fragmento posterior de 33 setígeros. Este consiste en seis pares de órganos copuladores dispuestos en parápodos sucesivos (Fig. 3A). Cirros ventrales de estos parápodos claramente diferentes al resto, largos, digitiformes y distalmente pilosos (Fig. 3B). Órganos copuladores distalmente curvados, con un abultamiento subdistal y extremo final bífido. Una papila filiforme ocasionalmente presente en una de estas dos ramas (Fig. 3C). 


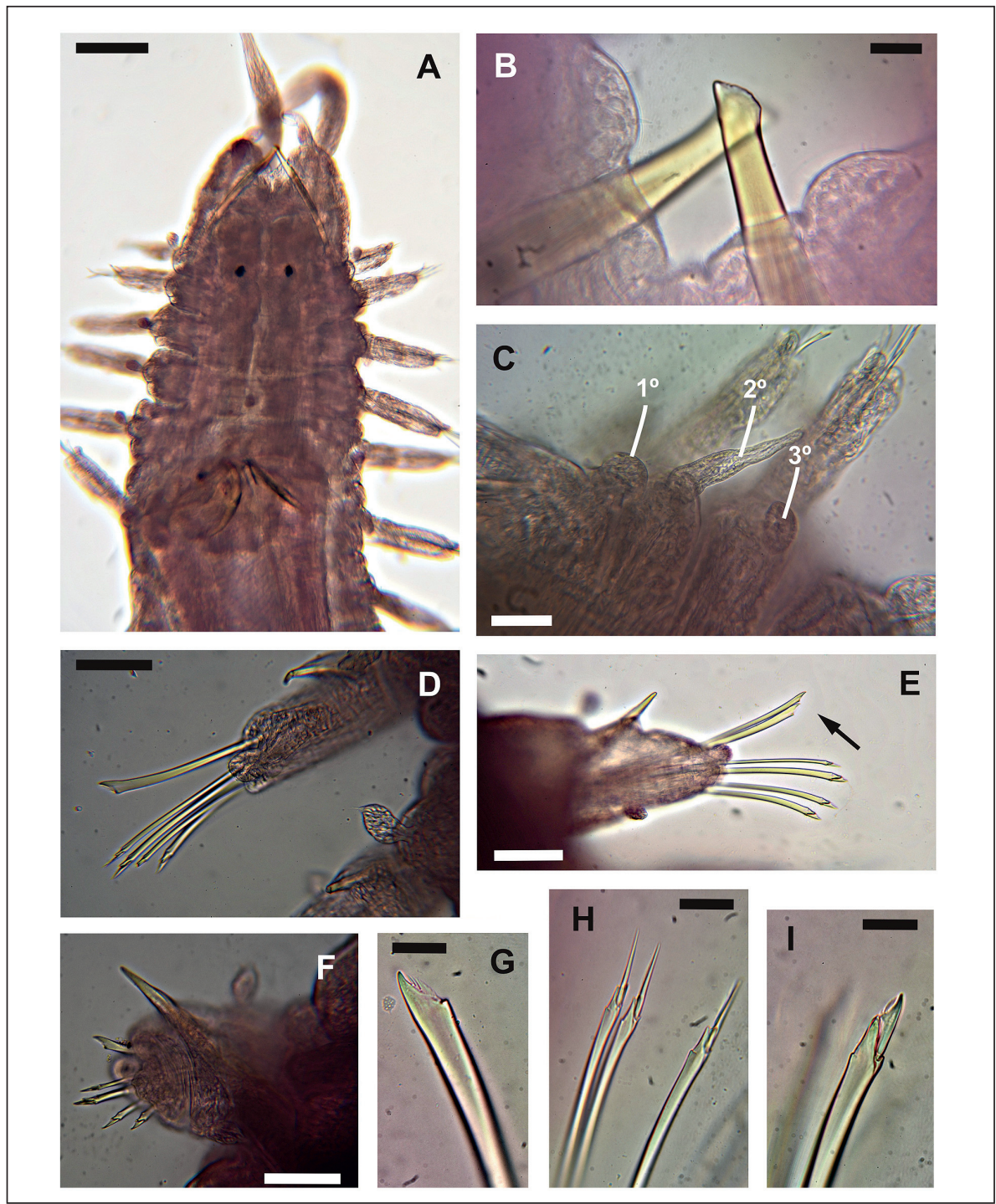

Fig. 2.- Pisione guanche San Martín, López \& Núñez, 1999. A: Extremo anterior, vista dorsal. B: Acículas bucales. C: Cirros dorsales de los tres primeros setígeros. D: Parápodo anterior, vista dorso-lateral. E: Parápodo posterior, vista lateral. F: Parápodo de la región media con dos sedas simples. G: Seda simple. H: Sedas compuestas, parápodo anterior. I: Seda compuesta, parápodo posterior. Escalas: $A=0,2 \mathrm{~mm} ; \mathrm{B}, \mathrm{G}, \mathrm{H}, \mathrm{I}=15 \mathrm{um} ; \mathrm{C}=50 \mathrm{um} ; \mathrm{D}, \mathrm{E}, \mathrm{F}=75 \mathrm{um}$.

Fig. 2.- Pisione guanche San Martín, López \& Núñez, 1999. A: Anterior end, dorsal view. B: Buccal aciculae. C: Dorsal cirri from the first three chaetigers. D: Anterior parapodium, dorsolateral view. E: Posterior parapodium, lateral view. F: Middle parapodium with two simple chaetae. G: Simple chaeta. H: Compound chaetae, anterior parapodium. I: Compound chaeta, posterior parapodium. Scales: $A=0.2 \mathrm{~mm} ; \mathrm{B}, \mathrm{G}, \mathrm{H}, \mathrm{I}=15 \mathrm{um} ; \mathrm{C}=50 \mathrm{um} ; \mathrm{D}, \mathrm{E}, \mathrm{F}=75 \mathrm{um}$. 


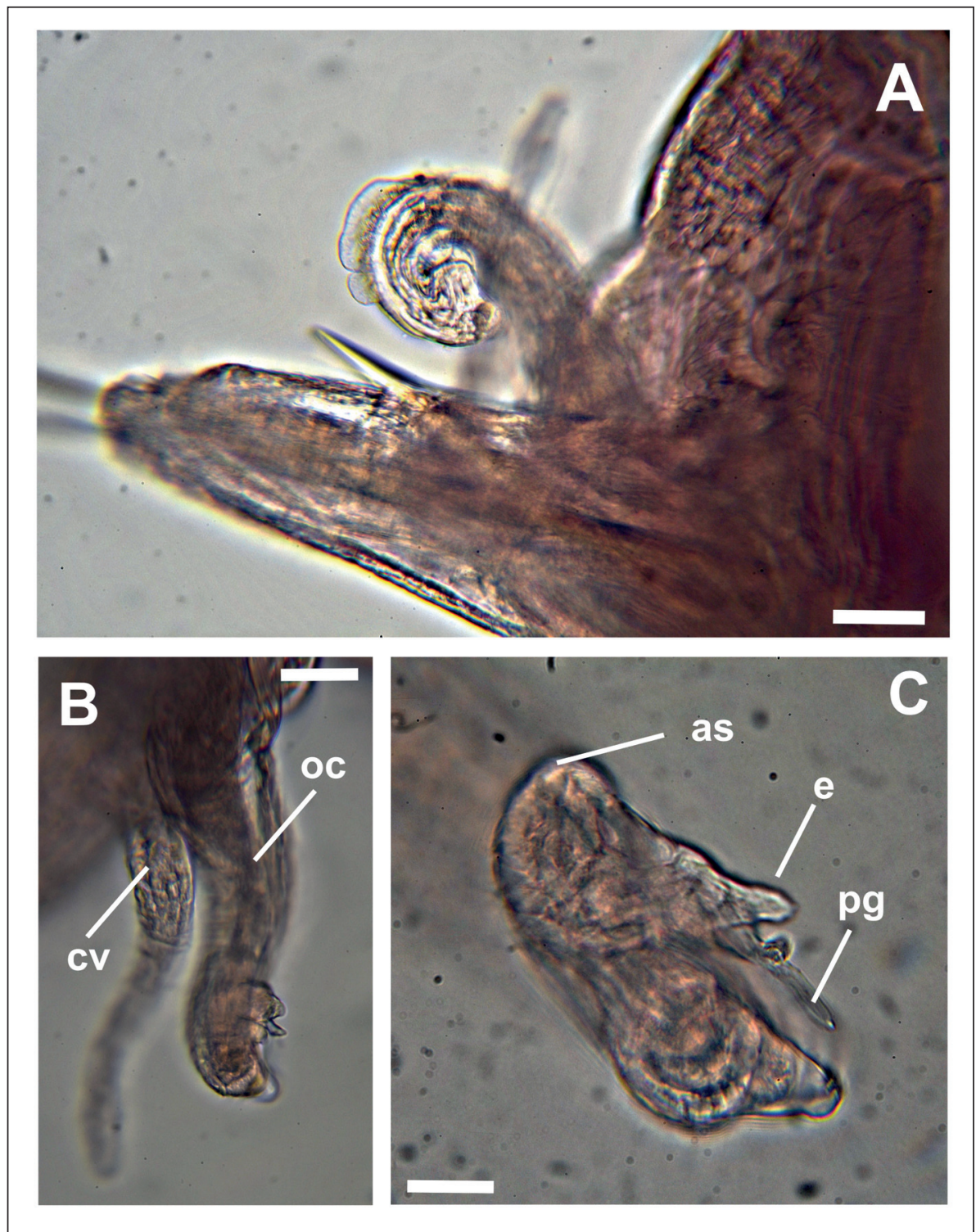

Fig. 3.- Pisione guanche San Martín, López \& Núñez, 1999. A: Parápodo modificado con órgano copulador. B: Extremo final de un órgano copulador masculino y cirro ventral alargado. C: Detalle del mismo. Abreviaturas: cv: cirro ventral; oc: órgano copulador; as: abultamiento subdistal; e: extremo bífido; pg: papila genital. Escalas: $A, B=50$ um; $C=25$ um.

Fig. 3.- Pisione guanche San Martín, López \& Núñez, 1999. A: Modified copulatory parapodium. B: Tip of a male copulatory organ and elongated ventral cirrus. C: Detail of the same.

Abbreviations: cv: ventral cirrus; oc: copulatory organ; as: subdistal bulk; e: bifid tip; pg: genital papilla. Scales: $A, B=50$ um; $C=25$ um. 


\section{Distribución}

Islas Canarias: Lanzarote, Fuerteventura, Gran Canaria, Tenerife, La Gomera y La Palma (Núñez et al, 2005). Mar de Alborán: Islas Chafarinas (Torres, 2008). Mar Levantino: Bahía de Iskenderun (Çinar, 2009). Islas Azores: San Miguel (Moreira et al., 2010). Plataforma continental de Portugal (Martins et al., 2012). Mar Cantábrico: Zarautz (este estudio).

\section{Ecología}

Fondos de gravas, arenas gruesas y arenas medias entre 8 y $80 \mathrm{~m}$ de profundidad (San Martín et al., 1999; Martins et al., 2012) y fondos de la fanerógama marina Cymodocea nodosa (Brito et al., 2005).

\section{Origen y vía de entrada}

La muestra donde se han obtenido los ejemplares de P. guanche corresponde a un fondo profusamente estudiado en las tres últimas décadas. Desde hace 26 años y con una periodicidad anual ha sido objeto de una exhaustiva caracterización biológica. En este periodo de tiempo se han realizado 546 dragados en este fondo y áreas próximas, siendo en los últimos dragados cuando ha tenido lugar su captura. Esta circunstancia, unida a la aparente expansión geográfica de este anélido hacia latitudes más septentrionales, nos lleva a interpretar su presencia en Zarautz como un nuevo caso de especie exótica en aguas del golfo de Vizcaya.

De hecho, en los últimos años se ha producido un notable incremento en el número de especies bentónicas exóticas que han llegado y establecido con éxito en el SE del golfo de Vizcaya, la mayoría de ellas procedentes de regiones más cálidas (Secilla et al., 1997; Ceberio et al., 1998; Martínez \& Adarraga, 2005, 2013; Martínez et al., 2006; Adarraga \& Martínez, 2011, 2012). Esto es debido, en parte, a las peculiaridades biogeográficas de esta zona. El mayor calentamiento que experimentan las capas superficiales del SE del golfo de Vizcaya respecto a las áreas geográficas adyacentes durante la época estival, favorece la existencia y aclimatación de especies de tendencia más meridional (Fischer, 1938; Ibáñez, 1989).

A día de hoy, no estamos en condiciones de determinar si la presencia de P. guanche en la costa de Zarautz es el producto de una introducción primaria o secundaria. El área donde la especie ha sido colectada carece de instalaciones de cultivos marinos próximas. En consecuencia, las vías de entrada más probables son el transporte naval o las corrientes marinas. La biología de este anélido (especie intersticial, de elevada movilidad y fases larvarias pelágicas) la hace propicia para su captación y traslado vía aguas de lastre; si bien la posesión de una fase larvaria planctónica de larga duración favorece también su traslado en las masas de agua circundantes (Åkesson, 1961). En este sentido, dicho autor señala que larvas de su congénere $P$. remota pueden permanecer alimentándose en la columna de agua hasta un periodo de 10 días en busca de un sustrato adecuado. Debido a ello, ambos mecanismos han sido postulados por otros autores como los principales vectores en la dispersión de ejemplares de Pisione (Gonzalez et al., 2017). 


\section{Agradecimientos}

Los autores del trabajo queremos agradecer a la Exma. Diputación Foral de Gipuzkoa por la financiación del presente Proyecto; a Iñaki Alberdi patrón de la embarción Oribay por la asistencia en el muestreo donde se obtuvo la especie; y finalmente, a los Drs. Julio Parapar de la Universidade da Coruña y Eduardo López de la Universidad Autónoma de Madrid por sus valiosos comentarios que han contribuido a mejorar el resultado final del artículo.

\section{Bibliografía}

- Adarraga, I., Martínez, J. 2011. First record of invasive Theora lubrica (Mollusca: Bivalvia: Semelidae) in the Atlantic Ocean. Mar. Biodivers. Rec. 4-e100: 1-7.

- Adarraga, I., Martínez, J. 2012. First record of the invasive brackish water mytilid Limnoperna securis (Lamarck, 1819) in the Bay of Biscay. Aquat. invasions 7(2): 171-180.

- Aguirrezabalaga, F., Altuna, A., Borja, A., Feliu, J., García-Carrascosa, A.M., Romero, A., San Vicente, C., Torres, J.A., Uriz, M.J., Ibáñez, M. 1984. Contribución al Conocimiento de la Fauna Marina de la Costa Vasca II. Lurralde 7: 83-133.

- Åkesson, B. 1961. On the histological differentiation of the larvae of Pisione remota (Pisionidae, Polychaeta). Acta Zoologica 42: 177-225.

- Brito, M.C. 1999. Estudio de las comunidades intersticiales del sebadal (Cymodocea nodosa) en Canarias con especial referencia a los Anélidos Poliquetos. Tesis Doctoral. Universidad de La Laguna. Tenerife.

- Brito, M.C., Martín, D., Núñez, J. 2005. Polychaetes associated to a Cymodocea nodosa meadow in the Canary Islands: assemblage structure, temporal variability and vertical distribution compared to other Mediterranean seagrass meadows. Mar. Biol. 146: 467-481.

- Ceberio, A., Martínez, J., Aguirrezabalaga, F. 1998. Presencia de Desdemona ornata Banse, 1957 (Polychaeta, Sabellidae) en las costas de la Península Ibérica, Golfo de Vizcaya. Munibe, cienc. Nat. 50: 37-41.

- Çinar, M.E. 2009. Alien polychaete species (Annelida: Polychaeta) on the southern coasts of Turkey (Levantine Sea, eastern Mediterranean), with 13 new records for the Mediterranean Sea. J. Nat. Hist. 43: 2283-2328.

- Fischer-Piette, E. 1938. Sur le caractère méridional du bios intercotidal du Golfe de Gascogne. C. R. Somm. Seances Soc. Biogeogr. 15(130): 61-65.

- Gonzalez, B.C., Petersen, H.C., Di Domenico, M., Martínez, A., Armenteros, M., García-Machado, E., Moller, P.R., Worsaae, K. 2017. Phylogeny and biogeography of the scaleless scale worm Pisione (Sigalionidae, Annelida). Ecol. Evol. 7(9): 2894-2915.

- Herrando-Pérez, S., San Martín, G., Núñez, J. 2001. Polychaete patterns from an oceanic island in the eastern Central Atlantic: La Gomera (Canary Archipelago). Cah. Biol. Mar. 42: 275-287. 
- Ibáñez, M. 1989. Implicaciones biogeográficas de la continentalización de la costa vasca. Lurralde 12: 71-101.

- Laubier 1967. Adaptations chez les Annélides Polychètes Interstitielles. Ann. Biol. 6: 1-16.

- Martínez, J., Adarraga, I. 2005. Programa de Vigilancia y Control de la Introducción de especies invasoras en los Ecosistemas Litorales de la Costa Vasca. 1. Costa de Gipuzkoa. Departamento de Medio Ambiente y Ordenación del Territorio, Gobierno Vasco. Vitoria-Gasteiz. Informe inédito.

- Martínez, J., Adarraga, I. 2013. First record of Paraprionospio coora Wilson, 1990 (Polychaeta: Spionidae) in the Atlantic Ocean. Bioinvasions Rec. 2(4): 271-280.

- Martínez, J., López, E., Adarraga, I. 2006. Nuevos datos del género Boccardia Carazzi, 1893 (Polychaeta: Spionidae) para la península Ibérica y el océano Atlántico. Bol. Inst. Esp. Oceanogr. 22(1-4): 53-64.

- Martínez, J., Aguirrezabalaga, F., Adarraga, I. 2008. A new species of Pisione (Annelida: Polychaeta: Pisionidae) from circalittoral soft bottoms (SE Bay of Biscay, Basque Coast). Cah. Biol. Mar. 49: 283-294.

- Martins, R., San Martín, G., Rodrigues, A.M., Quintino, V. 2012. On the diversity of the genus Pisione (Polychaeta, Pisionidae) along the Portuguese continental shelf, with a key to European species. Zootaxa 3450: 12-22.

- Moreira, J., Quintas, P. Troncoso, J.S. 2000. Pisione parapari n. sp., a new pisionid from the North-East Atlantic. Ophelia (Helsingør), 52(3): 177-182.

- Moreira, J., Veiga, P., Rubal, M. 2010. First record of Pisione guanche (Polychaeta: Pisionidae) at the Azores archipelago. Mar. Biodivers. Rec. 3 e-95: 1-6.

- Norlinder, E., Nygren, A., Wiklund, H., Pleijel, F. 2012. Phylogeny of scale-worms (Aphroditiformia, Annelida), assessed from 18SrRNA, 28SrRNA, 16SrRNA, mitochondrial cytochrome c oxidase subunit I (COI), and morphology. Phylogenetics Evol. 65: 490-500.

- Núñez, J., Brito, M.C., Docoito, J.M. 2005. Anélidos Poliquetos de Canarias: Catálogo de especies, distribución y hábitats. Vieraea 33: 297-321.

- Salcedo, D.L., Hernández-Alcántara, P., Solís-Weiss, V. 2015. Description of two new species of Pisione (Polychaeta: Sigalionidae) and first record of Pisione galapagoensis Westheide in the Southern Mexican Pacific. Zootaxa 4039(2): 373-390.

- San Martín, G. 2004. Familia Pisionidae Southern, 1914. En: Annelida, Polychaeta I. J.M. Viéitez, C. Alós, J. Parapar, C. Besteiro, J. Moreira, J. Núñez, J. Laborda, G. San Martín: 105-209. Museo Nacional de Ciencias Naturales. CSIC. Madrid. Fauna Ibérica 25.

- San Martin, G., López, E., Camacho, A.I, 1998. First record of a freshwater Pisionidae (Polychaeta): description of a new species from Panama with a key to the species of Pisione. J. Nat. Hist. 32: 1115-1127.

- San Martin, G., López, E., Núñez, J. 1999. Two new species of the genus Pisione Grube, 1857 from Cuba and the Canary Islands. Ophelia 51 (1): 29-38.

- Secilla, A., Gorostiaga, J.M., Díez, I., Santolaria, A. 1997. Amtithamnion amphigeneum (Ceramiales, Rhodophyta) from the European Atlantic Coasts. Botanica Mar. 40: 329-332. 
- Struck, T.H., Purschke, G., Halanych, K.M. 2005. A scaleless scale worm: Molecular evidence for the phylogenetic placement of Pisione remota (Pisionidae, Annelida). Mar. Biol. Res. 1:243-253.

- Torres, F.J. 2008. Estudio faunístico, ecológico y ambiental de la fauna de anélidos poliquetos de sustratos sueltos de las Islas Chafarinas (Mar de Alborán, S.W. Mediterráneo). Universidat de València. Valencia.

- Westheide, W. 1971. Interstitial Polychaeta (Excluding Archiannelida). Smithson. Contributions Zool. 76: 57-70.

- Wiklund, H., Nygren, A., Pleijel, F., Sundberg, P. 2005. Phylogeny of Aphroditiformia (Polychaeta) based on molecular and morphological data. Mol. Phylogenetics Evol. 37: 494-502.

- Yaminishi, R. 1998. Ten species of Pisione (Annelida: Polychaeta: Pisionidae) from Japan and evolutionary trends in the genus based on comparison of male copulatory apparatus. Publ. Seto Mar. Biol. Lab. 38: 83-145. 